\title{
Influencia de la temperatura en ensayos de arrancamiento sobre diferentes sistemas epoxi-acero de uso en conectores de ductos flexibles
}

\author{
Influence of temperature on pull out tests \\ on different steel-epoxy systems used in \\ end fittings of flexible pipes
}

Facundo Javier Wedekamper ${ }^{1}$, Diego Andrés Lorio ${ }^{1}$, Fabiano Bertoni ${ }^{2}$, Facundo Sebastián López ${ }^{2}$

\footnotetext{
${ }^{1}$ Programa de Pós-Graduação em Engenharia de Minas, Metalúrgica e de Materiais, UFRGS, CEP: 91501-970, Porto Alegre, Rio Grande do Sul - RS, Brasil.

e-mail: facundowedekamper@gmail.com; loriodiegoandres@gmail.com

${ }^{2}$ Símeros Technologies, Av. João Paris 739, CEP: 91160-440, Porto Alegre, Rio Grande do Sul - RS, Brasil. e-mail: bertoni@simeros.com; lopez@simeros.com
}

\section{RESUMEN}

En los yacimientos de petróleo offshore localizados en aguas profundas y ultra profundas son utilizados ductos flexibles. En muchos casos dentro de estos ductos los hidrocarburos son transportados a elevadas temperaturas. En los extremos de los ductos flexibles son instalados los conectores, siendo el vínculo entre el cuerpo del ducto y las plataformas o sistemas submarinos. En el montaje de los conectores, se utiliza resina epoxi para embeber los alambres de acero de la armadura de tracción y así formar el sistema de anclaje.

Los ensayos de arrancamiento permiten estudiar la interacción entre los materiales que forman el sistema de anclaje. El objetivo de este estudio es analizar bajo diferentes temperaturas la tensión de cizallamiento que tiende a separar la junta formada por la resina epoxi y el alambre de la armadura de tracción del ducto flexible. Para esto se realizaron ensayos de arrancamiento bajo dos temperaturas diferentes $\left(23^{\circ} \mathrm{C}\right.$ y $100^{\circ} \mathrm{C}$ ) en muestras confeccionadas con tres resinas epoxis diferentes. Se analizaron valores de tensión de cizallamiento en dos fases diferentes del ensayo, una de ellas es dependiente de la adhesión del polímero con el acero y la otra que es gobernada por la fricción entre los dos materiales. A través de análisis ANOVA para cada uno de los casos, se estableció que para las tres resinas evaluadas el incremento de la temperatura afecta los dos parámetros estudiados, disminuyendo su valor. Adicionalmente se evidenció que las resinas con carga mineral y de aluminio se vieron menos afectadas con respecto a la que no las poseía. Este estudio refleja la importancia de la apropiada selección y aplicación de los materiales epoxis utilizados en conectores, ya que una falla pone en riesgo el medio ambiente y la producción.

Palabras clave: Epoxi, Sistema de Anclaje, Ensayo de Arrancamiento, Temperatura.

\section{ABSTRACT}

In the offshore oil fields located in deep and ultra-deep waters they are used flexible pipes. In many cases inside these pipes, the hydrocarbons are transported at elevated temperatures. At the ends of the flexible pipes are installed end fittings, these are the connection with sea platforms and different subsea systems. In the assembly of end fittings, epoxy resin is used to embed the steel wires of the tensile armor and, in this way, form the anchorage system.

Pull out tests allow to study the interaction between the materials that form the anchoring system. The aim of this work is to analyze the shear stress which tends to separate the joint composed to the epoxy resin and wire armor tensile of flexible pipe under different temperatures. Thus pull out tests are performed under two different temperatures $\left(23^{\circ} \mathrm{C}\right.$ and $\left.100^{\circ} \mathrm{C}\right)$ in samples made with three different epoxies. Were analyzed two values of shear stress calculated in different phases of the test, one that is depen- 
dent on the polymer adhesion to steel, and the other is dependent on the friction between the materials. For each of the cases using ANOVA analysis, it was determinate that the temperature increase affects the two parameters studied decreasing its value and this is repeated for the three resins evaluated. Furthermore it was observed that the resins with inorganic and aluminum filler were less affected with respect which had not those fillers. This study show the importance of selection and application appropriate of epoxy materials used inside of the end fittings, because a failure is very dangerous to the environment and production.

Keywords: Epoxy, Anchoring System, Pull out Test, Temperature.

\section{INTRODUCCIÓN}

La resina epoxi es un material polimérico termoestable, que gracias a la flexibilidad en sus propiedades presenta una amplia gama de aplicaciones en diferentes ramas de la industria. Las variaciones en sus propiedades se obtienen a través de la incorporación de aditivos como diluyentes, flexibilizantes, cargas y pigmentos. Dentro de las características de los materiales epóxidos se destacan su alta resistencia mecánica, su estabilidad térmica, sus cualidades como adhesivo y su estabilidad química frente a diluyentes [2-3].

Los ductos flexibles en la actualidad son ampliamente utilizados en la explotación de hidrocarburos offshore, esto se debe a las ventajas que presenta su utilización en los yacimientos localizados debajo de láminas de agua profundas y ultra profundas. La resina epoxi es utilizada en la manufactura de los conectores, siendo estos, las terminaciones instaladas en los extremos de los ductos para vincularlos con las diferentes unidades de producción, como las plataformas en la superficie y dispositivos submarinos. Dentro de los conectores la resina epoxi embebe los alambres de acero que componen la armadura de tracción del ducto flexible, formando lo que se denomina el sistema de anclaje. Este sistema debe transmitir la mayor parte de las solicitaciones mecánicas provenientes del ducto, convirtiéndolo en un punto crítico para la integridad del sistema de producción [3-6].

En la operación de montaje del conector, una vez inyectada la resina epoxi dentro, la temperatura del sistema se eleva debido a la reacción exotérmica del proceso de cura. Cuando finaliza este proceso, el material epóxido se enfría con su consecuente contracción, este fenómeno trae como resultado una fuerza normal sobre el alambre de la armadura de tracción. La fuerza normal impuesta por la contracción térmica juega un rol importante en el sistema de anclaje [7].

En ciertas situaciones, las altas temperaturas en las cuales se extraen los hidrocarburos de los pozos profundos offshore traen beneficios, porque reduce la probabilidad de formación de depósitos sólidos de parafinas, hidratos y asfáltenos dentro de los ductos, estos compuestos pueden restringir u obstruir el paso del fluido [8]. Por otro lado, las elevadas temperaturas de boca de pozo podrían afectar el desempeño del sistema de anclaje porque, entre otras cosas, actúan sobre la fuerza normal producto del fenómeno de contracción térmica del polímero, influenciando en el comportamiento de la junta formada por el acero de la armadura de tracción y la resina epoxi, hecho que eventualmente pone en riesgo la integridad estructural de los conectores.

Los denominados ensayos de arrancamiento, son un método simplificado de estudiar en escala reducida el comportamiento de los elementos que forman el sistema de anclaje dentro de los conectores [6]. El objetivo de este trabajo es analizar la influencia del aumento de temperatura en los ensayos de arrancamiento, posibilitando un mayor entendimiento de la situación de los conectores cuando en servicio son expuestos a elevadas temperaturas.

\section{MATERIALES Y MÉTODOS}

\subsection{Delineamiento experimental}

El método de análisis a través de ensayos de arrancamiento, consiste básicamente en embeber un trozo de alambre de acero recto dentro de un cilindro de resina epoxi. Una vez sólida la resina, el alambre es arrancado del cilindro polimérico utilizando una máquina de tracción uniaxial, la cual impone un desplazamiento a velocidad constante [9]. La representación del sistema de anclaje a través de ensayos de arrancamiento permite evaluar la interacción entre el alambre de acero de la armadura de tracción y la resina epoxi, siendo una de las variables obtenidas del ensayo la fuerza que tiende a separar la junta. Para el estudio se propuso comparar los resultados obtenidos de ensayos de arrancamiento realizados a $23^{\circ} \mathrm{C}$ y $100^{\circ} \mathrm{C}$. 


\subsection{Preparación de muestras}

En este trabajo se evaluaron tres materiales epóxidos diferentes que son utilizados en conectores comerciales o experimentales. Los epoxis utilizados son del tipo bicomponente (resina/endurecedor), comercializados por la marca Huntsman y sus características principales son presentadas en la Tabla 1. Estos materiales son citados como "A", "B" $\mathrm{y}$ "C $\mathrm{C}$ " en este texto.

Tabla 1: Características de los sistemas epoxi utilizados.

\begin{tabular}{c|c|c|c}
\hline CARÁCTERÍSTICA & EPOXI A & EPOXI B & EPOXI C \\
\hline Nombre comercial de la resina & SW404 & AW4804 & XAW1395 \\
\hline Nombre comercial del endurecedor & HY404 & HW4804 & HY950 \\
\hline Proporción en peso de la mezcla & $10: 9$ & $100: 15$ & $100: 5$ \\
\hline Contenido de carga & Mineral & Aluminio & - \\
\hline
\end{tabular}

Las probetas se confeccionaron utilizando trozos de alambre rectos de armadura de tracción de ducto flexible, con dimensiones en la sección transversal de 2,5x $5 \mathrm{~mm}$, los cuales fueron embebidos en un cilindro de resina epoxi sólida de $30 \mathrm{~mm}$ de diámetro y una longitud de $65 \mathrm{~mm}$ como se ilustra en la Figura 1. La manufactura de las probetas se realizó mediante moldeo, utilizando las proporciones de resina/endurecedor establecidas por el fabricante. El proceso de cura se efectuó en un recinto cerrado bajo temperatura controlada durante $24 \mathrm{hs}$.

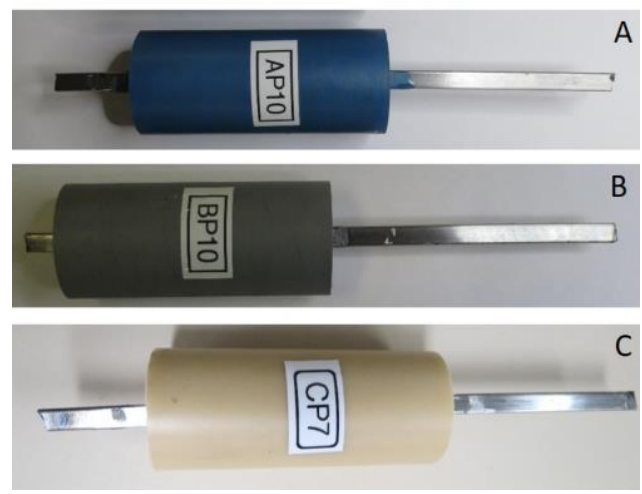

Figura 1: Probetas para ensayos de arrancamiento.

\subsection{Ensayos de arrancamiento}

Después de la preparación de las muestras, se realizaron los ensayos de arrancamiento utilizando una máquina de ensayos mecánicos MTS Landmark 370. El equipamiento fue ajustado para aplicar una velocidad de desplazamiento constante de $1 \mathrm{~mm} / \mathrm{min}$. Los parámetros registrados durante los ensayos fueron el desplazamiento y la fuerza resultante. En primera instancia, se ensayaron lotes de 12 muestras por cada tipo de resina evaluada a temperatura ambiente $\left(23^{\circ} \mathrm{C}\right)$.

Luego fue anexado un dispositivo de calefacción que posee un control automático de temperatura (Figura 2). Se realizaron pruebas utilizando 3 termopares (tipo K) adicionales al del sistema de control de calefacción, con los cuales se monitoreó las temperaturas en locales del recinto y dentro de la muestras, con esto se determinó el tiempo mínimo requerido a temperatura constante para que se homogenice el recinto y la muestra a $100^{\circ} \mathrm{C}$. Posteriormente fueron ensayadas 12 muestras más de cada resina epoxi en este valor de temperatura, manteniendo los demás parámetros iguales a los utilizados en los ensayos realizados a $23^{\circ} \mathrm{C}$. 


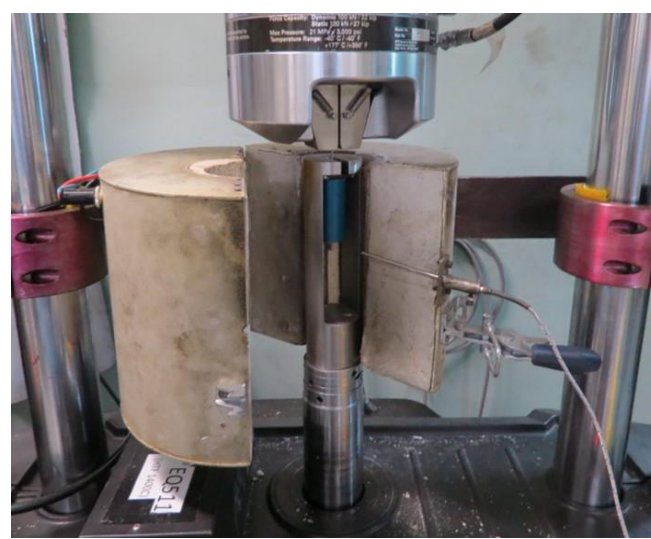

Figura 2: Ensayo de arrancamiento a alta temperatura.

\subsection{Análisis de datos}

A los fines de analizar los ensayos de arrancamiento y compararlos entre sí, se construyeron gráficos como el ilustrado en la Figura 3, donde el eje de las abscisas se representa el desplazamiento durante el ensayo y el eje de las ordenadas la tensión de cizallamiento que tiende a separar la junta formada por el alambre de acero y el material epoxi. Éste valor de tensión se obtiene al dividir la fuerza tractiva aplicada en el alambre por el área de su superficie que está en contacto con el polímero [6], utilizando este método de cálculo se asume que la tensión se distribuye de forma constante en el total de la superficie de alambre embebida en la matriz de resina. En diversos estudios, se ha probado que no necesariamente la tensión tiene una distribución uniforme en la región [10], pero esta consideración posibilita una simplificación eficaz para uniformizar los resultados y compararlos entre sí.

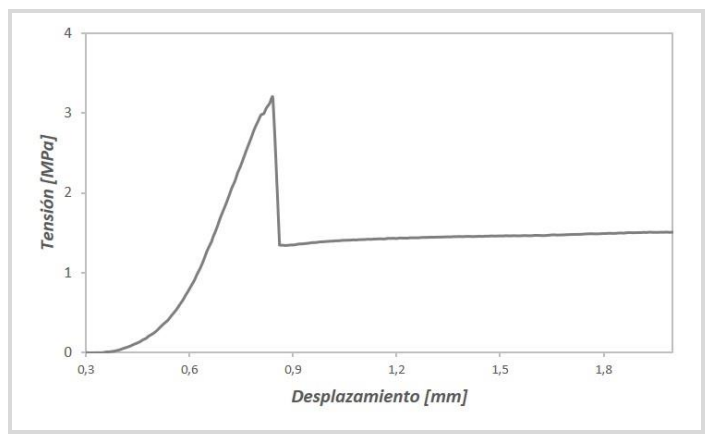

Figura 3: Curva típica de ensayo de arrancamiento.

Se pueden identificar tres regiones en las curvas de arrancamiento. En primera instancia una zona de comportamiento lineal, correspondiente con la elasticidad del polímero. La cual es seguida por un comportamiento no lineal, debido a que se ha sobrepasado el límite elástico del polímero, que se incrementa hasta un valor máximo. Finalmente, la carga cae bruscamente debido a que comienza a presentarse un desplazamiento relativo entre el alambre de acero y la resina epoxi [11]. En las curvas obtenidas, antes de comenzar el comportamiento elástico se observa una región no lineal producto principalmente a las holguras en los dispositivos de agarre, esta región no tiene consideración analítica. Entonces, es posible diferenciar dos fases en los ensayos de arrancamiento; en la primera fase no hay desplazamiento relativo entre la matriz de polímero y el alambre acero, predomina el comportamiento de adhesión de la resina con el substrato. La segunda fase se produce después del despegue del adhesivo con el substrato y es caracterizada por la fricción entre los materiales cuando se desplaza uno en relación al otro. Para el análisis se consideraron dos valores característicos de cada una de estas fases, el primero dado por la máxima tensión de adhesión y el segundo es el valor de la tensión producida por la fricción inmediatamente después del despegue. Siendo estos parámetros importantes en los proyectos de diseño de conectores [9].

Una vez determinadas la máxima tensión de adhesión y la tensión producida por la fricción en cada uno de los ensayos, se utilizó el análisis estadístico de varianzas denominado ANOVA (ANalysis Of VAriance) de un factor con un nivel de significancia de $95 \%$. Siendo el factor analizado la influencia de 
la temperatura sobre los ensayos de arrancamiento para cada resina epoxi. En el análisis, cuando $p<0,05$ se establece que los grupos son estadísticamente diferentes y por ende existe influencia de la temperatura.

\section{RESULTADOS}

Las medias y las desviaciones estándar calculadas de la máxima tensión de adhesión y la tensión producida por la fricción obtenida en cada grupo de ensayos de arrancamiento son expuestos en los gráficos de la Figura 4 y la Figura 5.

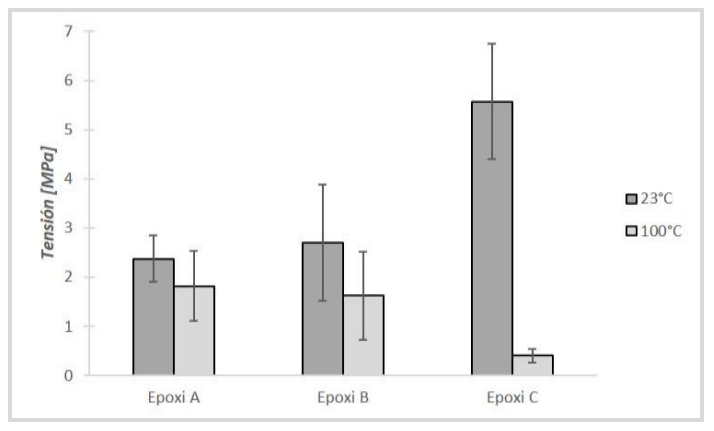

Figura 4: Tensión máxima de adhesión.

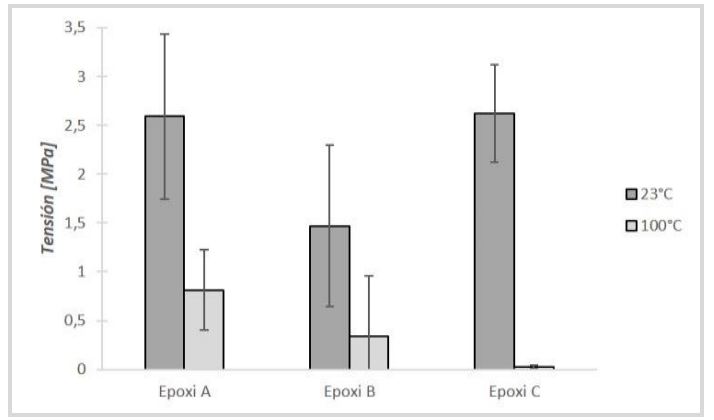

Figura 5: Tensión producto de la fricción.

El análisis ANOVA sobre los valores obtenidos determinó que existen diferencias estadísticamente significativas tanto para la tensión máxima de adhesión entre la superficie del polímero y el acero, como para la tensión producto de la fricción entre estos materiales, y esto ocurre para las tres resinas epoxis analizadas. Siendo así es posible afirmar que la temperatura afecta los resultados de los ensayos de arrancamiento disminuyendo los valores de tensiones obtenidas.

Para comparar la influencia que tiene la temperatura sobre los ensayos de arrancamiento entre las tres diferentes resinas epoxis estudiadas, se determinó el porcentaje de disminución de las medias de tensión analizadas, para lo cual se utilizó la ecuación siguiente:

$$
\Delta \tau_{\%}=\frac{\tau_{23^{\circ} \mathrm{C}}-\tau_{100^{\circ} \mathrm{C}}}{\tau_{23^{\circ} \mathrm{C}}} \times 100
$$

Donde $\Delta \tau \%$ es el porcentaje de disminución de tensión, $\tau 23^{\circ} \mathrm{C}$ es la tensión obtenida a partir de los ensayos realizados a $23^{\circ} \mathrm{C}$ y $\tau 100^{\circ} \mathrm{C}$ es la tensión obtenida a partir de los ensayos realizados a $100^{\circ} \mathrm{C}$. Los valores resultantes se exponen en la Tabla 2.

Tabla 2: Cociente entre las medias de las tensiones evaluadas a diferentes temperaturas.

\begin{tabular}{c|c|c|c}
\hline TENSIÓN ANALIZADA & EPOXI A & EPOXI B & EPOXI C \\
\hline Máxima de adhesión & $24 \%$ & $40 \%$ & $93 \%$ \\
\hline De fricción & $69 \%$ & $77 \%$ & $99 \%$ \\
\hline
\end{tabular}




\section{DISCUSIÓN}

La tensión máxima de adhesión depende principalmente de características físico-químicas de los componentes de la junta, así como de la tensión superficial del adhesivo y del substrato, la polaridad y la interface de las superficies [2]. Estas propiedades se ven afectadas por los aditivos incorporados en el adhesivo epoxi, en la resina A sobresale las carga mineral que posee, mientras que en la B la carga de aluminio, para la resina $\mathrm{C}$ no se especifican cargas de este tipo [12-14]. Las cargas en algunos casos pueden llegar a suponer hasta el $80 \%$ del producto, estas son introducidas principalmente para reducir su costo y/o para mejorar alguna propiedad específica.

Como el coeficiente de expansión térmica del sustrato (acero) y el adhesivo (epoxi) es diferente, cuando la junta formada por estos dos materiales es sometida a una variación de temperatura se generan tensiones internas que tienden a disminuir la tensión que es capaz de soportar la junta [15]. La incorporación de cargas al sistema epoxi disminuye el coeficiente de expansión térmica del adhesivo, aproximando su valor al del acero, disminuyendo el efecto de las tensiones internas generadas por la variación de temperatura [15]. La presencia de las cargas en la resina A y B explica la menor influencia del aumento de temperatura sobre la tensión máxima de adhesión en estas resinas, con respecto a la resina $\mathrm{C}$, como se ve reflejado en el menor porcentaje de disminución de tensión media de A y B en referencia a C.

Cuando se produce el despegue de la junta, la tensión en el ensayo de arrancamiento se vuelve dependiente de la fricción entre las dos superficies debido a que existe desplazamiento relativo entre ellas. Es sabido que la fricción depende del coeficiente de fricción entre los materiales y de la fuerza normal aplicada. La dilatación térmica de la resina epoxi es mayor a la del acero del alambre, entonces es de esperarse que la fuerza normal disminuya con el aumento de la temperatura, provocando la disminución de la fuerza producto de la fricción; lo que se refleja en los valores medios en el gráfico de la Figura 5, donde para las tres resinas epoxis evaluadas el valor medio de tensión se redujo. La incorporación de cargas, tanto mineral como de aluminio, afecta el coeficiente de dilatación térmica del polímero, disminuyéndolo, lo que se traduce en menos expansión térmica y, por lo tanto, menos influencia del incremento de temperatura sobre los resultados del ensayo. Fue observado un menor porcentaje de disminución de la tensión producto de la fricción tanto en la resina $\mathrm{A}$, como en la resina $\mathrm{B}$ con respecto a la $\mathrm{C}$ que no contiene estas cargas.

\section{CONCLUSIONES}

El desempeño de tres resinas epoxi fue evaluado en ensayos de arrancamiento, orientados al análisis de sistemas de anclaje para ductos flexibles. Este tipo de ensayos son un modo económico y simple de evaluar la performance del sistema de anclaje compuesto por la armadura de tracción y la resina termoestable epoxi. Los ensayos se realizaron primero a $23^{\circ} \mathrm{C}$ y luego a $100^{\circ} \mathrm{C}$ con el objetivo de determinar la influencia del incremento de temperatura, siendo la tensión máxima de adhesión y la tensión producto de la fricción, las variables analizadas. Comparando los resultados de los ensayos a $23^{\circ} \mathrm{C}$ con los que se realizaron a $100^{\circ} \mathrm{C}$ se observó una disminución estadísticamente significativa de los dos parámetros estudiados, el fenómeno se repite para las tres resinas evaluadas, evidenciando que el incremento de temperatura afecta al desempeño de los materiales en los ensayos de arrancamiento, resultado que podría extrapolarse al sistema de anclaje de los ductos flexibles. Adicionalmente, se observó que tanto la resina epoxi que posee carga mineral, como la que incorpora carga de aluminio, fueron menos afectadas por el incremento de la temperatura en los parámetros estudiados.

Las altas temperaturas de servicio a las que pueden ser sometidos los conectores, pueden afectar el desempeño de su sistema de anclaje, hecho que debe considerarse en el proyecto de diseño de este componente ya que su integridad estructural es crítica para tanto en la producción como para evitar posibles incidentes con sus eventuales consecuencias, tanto ambientales como económicas.

\section{AGRADECIMIENTOS}

Los autores de este trabajo quieren agradecer al Laboratório de Metalurgia Física LAMEF/UFRGS por el apoyo y por brindar sus instalaciones para realizar los ensayos. 


\section{BIBLIOGRAFÍA}

[1] MUDRY, G. A., Revestimentos de silanos obtidos sobre aço carbono e sua Utilização como agentes promovedores de adesão em uniões aço-resina epóxi, Tesis de M.Sc., PPGE3M/UFRGS, Porto Alegre, RS, Brasil, 2011.

[2] DAFLON, M. B., Estudo de adhêrencia de arames da armadura de tração em conectores de dutos flexíveis, Tesis de M.Sc., UERJ/PPGCTM, Nova Friburgo, RJ, Brasil, 2010.

[3] API 17B, Recomended Practice for Flexible Pipe, 2002.

[4] CAMPELlO, G. C., CARPIGIANI, M., BERTONI, F., et al., "A Novel Concept of Flexible Pipe End Fitting: Tensile Armor Foldless Assembly", In: Proceedings of the ASME 201231 st International Conference on Ocean, Offshore and Arctic Engineering, OMAE2012-8351, Rio de Janeiro, p. 1, July 2012.

[5] XAVIER, F. G., Avaliação da vida em fadiga de um novo modelo de conector para dutos flexíveis Tesis de D.Sc., PPGE3M/UFRGS, Porto Alegre, RS, Brasil, 2009.

[6] TORRES, R.N., TANAKA, R. L., MORINI, R. G., et al., "Flexible pipe anchoring system: resin ratio effects on mechanical properties", In: Proceedings of the ASME 2015 34th International Conference on Ocean, Offshore and Arctic Engineering, OMAE2015-41284, St. John's, Newfoundland, Canada, p.p. 1-2, May-June 2015.

[7] OLAF, O., TANAKA, R. L., MORINI, R. G., et al., "Thermal effects on the anchoring of flexible pipe tensile armors", In: Proceedings of the ASME 2015 34th International Conference on Ocean, Offshore and Arctic Engineering, OMAE2015-41284, St. John's, Newfoundland, Canada, p. 2, May-June 2015.

[8] MORAIS, J. M., Petróleo em águas profundas: uma história tecnológica da PETROBRAS na exploração e produção offshore, 1 ed., Brasília, Instituto de Pesquisa Econômica Aplicada, 2013.

[9] CAMPELLO, G. C., Metodologia de projeto para o sistema de ancoragem de conectores de dutos flexiveis e proposição de nova tecnologia, Tesis de D.Sc., COPPE/UFRJ, Rio de Janeiro, RJ, Brasil, pp. 46-48, 2014.

[10] HSUEH, C.-H., "Interfacial Debonding and Pull-Out Stresses of Fiber-Reinforced Composites VII: Improved Analysis for Bonded Interfaces", Materials Science and Engineering A, v. 154, pp. 125-132, July 1992.

[11] HSUEH, C.-H., "Interfacial Debonding and Fiber Pull-Out Stresses of Fiber-Reinforced Composites", Materials Science and Engineering A, v. 132, pp. 1-11, July 1989.

[12] HUNTSMAN ADVANCED MATERIALS, “RenGel® SW 404 / Ren® HY 404”, 2010.

[13] HUNTSMAN ADVANCED MATERIALS, “Araldite® AW 4804 / Hardener HW 4804”, , 2009.

[14] HUNTSMAN ADVANCED MATERIALS, “Araldite XAW 1395 BZ/ Endurecedor HY 950”, 2007.

[15] PETRIE, E. M., Epoxy Adhesive formulations, 1 ed., New York, McGraw-Hill, 2006. 